\title{
Endocrinopatias Associadas ao Tratamento com Inibidores do Checkpoint Imunológico
}

\section{Endocrinopathies Associated with Immune Checkpoint Inhibitors}

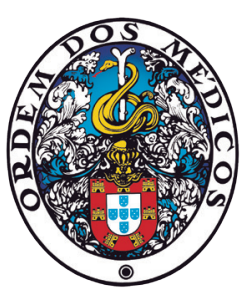

Maria Joana SANTOS $\square 1$

Acta Med Port 2022 Mar;35(3):209-215 - https://doi.org/10.20344/amp.13673

RESUMO

Introdução: Os inibidores do checkpoint imunológico (ICl) são anticorpos monoclonais que permitem aumentar a eficiência do sistema imunitário na destruição das células neoplásicas. Nos últimos anos, estes fármacos têm sido cada vez mais utilizados no tratamento de muitas neoplasias em estadios avançados. Contudo, a alteração da regulação do sistema imunitário induzida por estes fármacos tem como potencial efeito adverso o aparecimento de autoimunidade em praticamente todos os órgãos. As endocrinopatias são um dos eventos adversos autoimunes mais frequentes com estes fármacos.

Material e Métodos: Revisão não sistemática sobre as endocrinopatias reportadas em contexto de tratamento com ICI. Foram pesquisados artigos publicados na PubMed até 31 de janeiro de 2020, selecionados com base na sua relevância e excluídos em caso de conteúdo redundante. Foram utilizados os seguintes termos de pesquisa: "immune checkpoint inhibitor" e "endocrinopathy" / "endocrine system diseases" / "pituitary" / "thyroid" / "diabetes" / "adrenal" / "parathyroid".

Resultados: Foram já reportadas endocrinopatias com todas as classes de ICl (anti-CTLA-4, anti-PD-1, anti-PD-L1). A disfunção tiroideia é a endocrinopatia mais frequentemente reportada, principalmente sob anti-PD-1 e anti-PD-L1. A hipofisite é a mais prevalente sob anti-CTLA-4. É crescente a incidência de diabetes autoimune neste contexto, principalmente sob anti-PD-1 e anti-PD-L1. Foram reportados também casos raros de insuficiência suprarrenal primária, doença de Graves e hipoparatiroidismo primário.

Conclusão: O conhecimento do espectro de endocrinopatias desencadeadas pela terapêutica com ICl, assim como as suas manifestações clínicas, critérios de diagnóstico e tratamento, é essencial, dada a sua elevada prevalência e o cada vez maior número de doentes oncológicos tratados com estes novos fármacos.

Palavras-chave: Antineoplásicos Imunológicos; Doenças do Sistema Endócrino; Inibidores de Checkpoint Imunológico; Receptor de Morte Celular Programada 1

\section{ABSTRACT}

Introduction: Immune checkpoint inhibitors (ICls) are monoclonal antibodies that increase the efficiency of the immune system in the destruction of neoplastic cells. In recent years, these drugs have been increasingly used in the treatment of many neoplasms in advanced stages. However, the change in the regulation of the immune system induced by these drugs has the potential adverse effect of inducing autoimmunity in practically all organ systems. Endocrinopathies are one of the most common autoimmune adverse events of these drugs.

Material and Methods: Non-systematic review of endocrinopathies reported in the context of treatment with ICls. A search was carried out on PubMed until January 31 ${ }^{\text {st }}, 2020$, and articles were selected based on their relevance and excluded in case of redundant content. The following search terms were used: "immune checkpoint inhibitor" and "endocrinopathy" / "endocrine system diseases" / "pituitary" / "thyroid" / "diabetes" / "adrenal" / "parathyroid".

Results: Endocrinopathies with all classes of ICIs (anti-CTLA-4, anti-PD-1, anti-PD-L1) have been reported. Thyroid dysfunction is the most frequently reported endocrinopathy, mainly with anti-PD-1 and anti-PD-L1. Hypophysitis is the most prevalent with anti-CTLA-4. The incidence of autoimmune diabetes in this context is increasing, mainly with anti-PD-1 and anti-PD-L1. Rare cases of primary adrenal insufficiency, Graves' disease and primary hypoparathyroidism have also been reported.

Conclusion: Knowing the spectrum of endocrinopathies triggered by $\mathrm{ICl}$, as well as their clinical features, diagnosis and treatment criteria is essential, given its high prevalence and the increasing number of cancer patients treated with these new drugs.

Keywords: Antineoplastic Agents, Immunological; Endocrine System Diseases; Immune Checkpoint Inhibitors; Programmed Cell Death 1 Receptor

\section{INTRODUÇÃO}

Os checkpoint imunológicos são mecanismos essenciais para a regulação da resposta do sistema imunitário a antigénios estranhos. Podem ser estimuladores ou inibidores, levando à destruição de antigénios estranhos ao organismo ou supressão da resposta autoimune. ${ }^{1}$ Muitas células neoplásicas conseguem induzir o aumento da expressão de checkpoint imunológicos inibitórios, o que lhes permite evadirem-se da ação antitumoral das células T citotóxicas. A descoberta deste mecanismo de sobrevivência das células tumorais, revolucionou o tratamento de muitas neoplasias, levando ao desenvolvimento de uma nova clas-

1. Serviço de Endocrinologia. Hospital de Braga. Braga. Portugal.
$\square$ Autor correspondente: Maria Joana Santos. mjoanasantos@hotmail.com

Recebido: 02 de março de 2020 - Aceite: 16 de setembro de 2020 - Online issue published: 25 de janeiro de 2022 - Online issue published: 02 de março de 2022 Copyright $\odot$ Ordem dos Médicos 2022

se farmacológica: os inibidores do checkpoint imunológico $(\mathrm{ICl}){ }^{1}$

\section{Inibidores do checkpoint imunológico}

Os inibidores do checkpoint imunológico são anticorpos monoclonais que bloqueiam a atividade inibitória dos mecanismos de resposta imunológica a antigénios tumorais, reativando desta forma os linfócitos T citotóxicos contra as células tumorais. ${ }^{1} \mathrm{Os} \mathrm{ICl}$ não atuam diretamente nas células tumorais, mas nos recetores dos linfócitos ou seus ligandos, estimulando a atividade destas células. ${ }^{1}$ Têm um 
efeito antitumoral eficaz e duradouro num amplo espectro de neoplasias em estadio avançado e têm tido uma utilização crescente desde a aprovação do primeiro fármaco da classe, em 2011. ${ }^{1-3}$

Existem, atualmente, três classes de $\mathrm{ICl}$ aprovadas pela Food \& Drug Administration (FDA) e pela Agência Europeia do Medicamento (EMA): os anti-CTLA-4, os anti-PD-1 e os anti-PD-L1 (Tabela 1). ${ }^{4}$

O CTLA- 4 (cytotoxic T-lymphocyte-associated protein 4) inibe a ativação precoce das células $T$ citotóxicas nos gânglios linfáticos, diminui a atividade cas células $\mathrm{T}$ helper e aumenta a atividade imunossupressora das células $T$ reguladoras. ${ }^{1-3} \mathrm{O}$ seu bloqueio impede a indução de anergia das células T, aumentando a atividade das células T CD8+ e CD4+ efetoras e inibindo a ação das células T reguladoras. ${ }^{1,2} \mathrm{O}$ primeiro $\mathrm{ICl}$ aprovado pela FDA, em 2011, foi o ipilimumab, um anti-CTLA-4.

O PD-1 (programmed cell death protein 1) é expresso numa maior variedade de células: células $T$ citotóxicas, células $T$ reguladoras, células $B$, células natural killer, macrófagos e células dendríticas. ${ }^{1,2}$ Limita a atividade pró-inflamatória destas células e previne a autoimunidade. No microambiente tumoral, a ativação da via do PD-1 é um importante mecanismo de escape das células tumorais à destruição pelo sistema imunitário. A exposição crónica dos linfócitos T a antigénios tumorais promove a expressão persistente de PD-1 nos linfócitos e a sua anergia/exaustão. ${ }^{1,2}$ A segunda classe de ICls aprovada foi a dos anti-PD-1. Ao bloquearem a via PD-1, estes fármacos aumentam a ação das células $T$ efetoras e diminuem a ação das células $T$ reguladoras nos tecidos e microambiente tumoral.

O PD-1 possui dois ligandos (PD-L1 e PD-L2). O PD-L1 é expresso em muitos tipos de células linfóides e não linfóides, sendo um importante mecanismo de proteção contra a autoimunidade. ${ }^{2}$ O PD-L2 é principalmente expresso nas células apresentadoras de antigénios e diminui a resposta imune induzida pelas células Th2. ${ }^{2}$ O PD-L1 e o PD-L2 existem em grande quantidade à superfície de muitos tumores. Através deles, as células tumorais ligam-se aos recetores de PD-1 na superfície dos linfócitos, levando à inativação dos linfócitos T. Os anti-PD-L1, anticorpos monoclonais que se ligam aos ligandos do PD-1 na superfície das células tumorais, foram a terceira classe de $\mathrm{ICl}$ aprovada pela FDA (Tabela 1). ${ }^{4}$

\section{MATERIAL E MÉTODOS}

Foi feita uma revisão não sistemática sobre endocrinopatias em contexto de tratamento com ICl. Para esse efeito, foi feita uma pesquisa na PubMed de todos os artigos publicados até 31 de janeiro de 2020, tendo por base as seguintes palavras-chave: "immune checkpoint inhibitor" e "endocrinopathy" / "endocrine system diseases" / "pituitary" / "thyroid" / "diabetes" / "adrenal" / "parathyroid". Todos os artigos foram lidos e posteriormente selecionados com base na sua relevância. Foram incluídos todos os tipos de artigos, incluindo relatos de caso. Foram excluídos artigos cujo conteúdo fosse considerado redundante.

\section{RESULTADOS}

\section{Eventos adversos autoimunes por $\mathrm{ICI}$}

A desinibição do sistema imunitário obtida pelo bloqueio de vias inibitórias dos checkpoint imunológicos tem um potencial inerente de autoimunidade. ${ }^{2}$ Foram já descritos numerosos eventos adversos autoimunes em contexto de

Tabela 1 - Inibidores do checkpoint aprovados pela FDA 4

\begin{tabular}{|c|c|c|c|}
\hline Classe de ICl & Fármaco & Ano de provação & Indicações aprovadas \\
\hline Anti-CTLA-4 & Ipilimumab & 2011 & $\begin{array}{l}\text { melanoma; carcinoma de células renais; carcinoma colorrectal com ins- } \\
\text { tabilidade de microssatélite }\end{array}$ \\
\hline \multirow[t]{3}{*}{ Anti-PD-1 } & Pembrolizumab & 2014 & $\begin{array}{l}\text { melanoma; carcinoma do pulmão de não pequenas células; carcinoma } \\
\text { do pulmão de pequenas células; carcinoma espinocelular da cabeça/ } \\
\text { pescoço; linfoma de Hodgkin clássico; linfoma primário do mediastino } \\
\text { de grandes células B; carcinoma urotelial; neoplasias com elevada ins- } \\
\text { tabilidade de microssatélite; carcinoma gástrico; carcinoma do esófago; } \\
\text { carcinoma do colo do útero; carcinoma hepatocelular; carcinoma de cé- } \\
\text { lulas de Merkel; carcinoma de células renais; carcinoma do endométrio }\end{array}$ \\
\hline & Nivolumab & 2014 & $\begin{array}{l}\text { melanoma, carcinoma pulmão de não pequenas células; carcinoma do } \\
\text { pulmão de pequenas células; carcinoma espinocelular da cabeça/pesco- } \\
\text { ço; linfoma de Hodgkin clássico; carcinoma de células renais; carcinoma } \\
\text { urotelial; carcinoma colorrectal com elevada instabilidade de microssaté- } \\
\text { lite; carcinoma hepatocelular }\end{array}$ \\
\hline & Cemiplimab & 2018 & carcinoma espinocelular \\
\hline \multirow[t]{3}{*}{ Anti-PD-L1 } & Atezolizumab & 2016 & $\begin{array}{l}\text { carcinoma urotelial; carcinoma do pulmão de não pequenas células; } \\
\text { carcinoma do pulmão de pequenas células; carcinoma da mama triplo } \\
\text { negativo }\end{array}$ \\
\hline & Durvalumab & 2017 & carcinoma pulmão de não pequenas células; carcinoma urotelial \\
\hline & Avelumab & 2017 & $\begin{array}{l}\text { carcinoma urotelial; carcinoma de células de Merkel; carcinoma de cé- } \\
\text { lulas renais }\end{array}$ \\
\hline
\end{tabular}

FDA: Food and Drug Administration; ICl: inibidor do checkpoint imunológico; CTLA-4: antigénio-4 de linfócito T citotóxico; PD-1: proteína de morte celular programada 1; PD-L1: ligando 1 da proteína de morte celular programada 1 
$\mathrm{ICl}$ [efeitos adversos imunorrelacionados (EAir)]. Os EAir podem atingir qualquer órgão com uma gravidade variável e causar inclusivamente morte/risco de morte. ${ }^{2} \mathrm{O}$ tratamento combinado com duas classes de $\mathrm{ICl}$ associa-se a maior incidência e gravidade de EAir. ${ }^{2,5}$ Estes eventos adversos podem surgir a qualquer momento após o início do tratamento, até meses depois da sua suspensão. ${ }^{5}$ Vários estudos sugerem a existência de uma associação positiva entre a ocorrência de EAir e maior resposta tumoral ao tratamento, com melhor prognóstico da doença oncológica, o que traduz uma maior capacidade do sistema imunitário em reconhecer e destruir as células neoplásicas. No entanto, esta associação não está claramente provada. ${ }^{2}$

\section{Endocrinopatias associadas a ICI}

As glândulas endócrinas são dos órgãos mais frequentemente afetados por EAir em contexto de ICI. Numa meta-análise com 38 ensaios clínicos e 7551 doentes, a incidência global de endocrinopatias foi de $6,6 \% .^{6}$ Contudo, estudos mais recentes apontam para uma incidência entre $4 \%-30 \% .{ }^{7}$ Dados da vida real, obtidos após a introdução dos fármacos no mercado, têm mostrado uma incidência crescente do número de endocrinopatias descritas em contexto de $\mathrm{ICI}$, principalmente nos primeiros quatro anos após a sua comercialização. ${ }^{8,9}$ No maior estudo de farmacovigilância sobre endocrinopatias por ICls publicado até à data, com mais de 6000 doenças endócrinas registadas, verificou-se a ocorrência de $54,5 \%$ sob anti-PD-1; o risco de endocrinopatia foi maior sob anti-CTLA-4; a terapêutica combinada de anti-PD-1 e anti-CTLA-4 associou-se a um maior risco e maior frequência destes EAir; $17 \%$ das endocrinopatias associaram-se a morte/risco de morte, provavelmente por dificuldade/atraso na sua deteção e tratamento atempados. ${ }^{8,10}$ É necessário um elevado grau de suspeição para o diagnóstico de endocrinopatias por $\mathrm{ICI}$, pois alguns dos seus sintomas mais comuns (astenia, anorexia, emagrecimento) são frequentemente observados em doentes oncológicos. ${ }^{11}$

\section{Hipofisite}

A hipofisite foi das primeiras endocrinopatias descritas em contexto de $\mathrm{ICl}$ e estima-se que atinja cerca de $3 \%$ dos doentes. ${ }^{12}$ Pode aparecer em qualquer momento após o início do tratamento. ${ }^{2,13} \mathrm{O}$ seu mecanismo patofisiológico é desconhecido e parece não depender da existência de autoimunidade hipofisária prévia. ${ }^{13}$ Foi documentada necrose extensa da adenohipófise com ausência total de gonadotrofos e tireotrofos na única autópsia disponível. ${ }^{14}$ Foram propostos dois mecanismos fisiopatológicos: ativação da via clássica do complemento e citotoxicidade mediada por células e dependente de anticorpos (ADCC). O risco de hipofisite parece depender de fatores individuais (polimorfismos na expressão de CTLA-4 na hipófise) e também da classe de ICI (diferente potência relativa para ativar complemento e ADCC). ${ }^{2,15}$ Apesar de já ter sido relacionada com as três classes de $\mathrm{ICl}$, a endocrinopatia é mais frequentemente associada aos anti-CTLA-4, com risco dependente da dose, sobretudo em terapêutica combinada. ${ }^{2,6,15-17} A$ maior série de hipofisites por ICl publicada até à data, com dados da vida real, incluiu 94 casos em 249 endocrinopatias $(37,8 \%$ das endocrinopatias reportadas). O fármaco mais associado com hipofisite foi o ipilimumab (43\%), mas a incidência com anti-PD-1 foi semelhante (nivolumab 30\%, pembrolizumab 14\%), mostrando que esta endocrinopatia pode ocorrer com qualquer classe de $\mathrm{ICl}$. O tempo de tratamento até à hipofisite foi menor com ipilimumab (em monoterapia ou associação) do que com anti-PD-1 em monoterapia. A gravidade da hipofisite foi menor com pembrolizumab do que com nivolumab e ipilimumab. ${ }^{13}$

A apresentação clínica consiste em sinais/sintomas inespecíficos devido ao hipopituitarismo, sendo os mais frequentes astenia e cefaleias. Os doentes podem também apresentar náuseas, anorexia, tonturas, hipotensão, diminuição da líbido, intolerância ao frio, emagrecimento, sintomas psiquiátricos (apatia, ansiedade, depressão), febre, hipoglicemia ou dores articulares. ${ }^{2,11}$ Pode ocorrer efeito de massa por aumento do volume da hipófise, mas é geralmente ligeiro e transitório. ${ }^{2}$ As células hipofisárias mais frequentemente atingidas são os corticotrofos [produtores de hormona adrenocorticotrófica (ACTH)], os tirotrofos [produtores de hormona tiroestimulante (TSH)] e os gonadotrofos [produtores de hormona luteinizante (LH) e de hormona folículo-estimulante (FSH)]. O défice de ACTH está presente em quase todos os doentes, é geralmente persistente e o maior responsável pela morbilidade associada a este tipo de hipofisite. ${ }^{2} \mathrm{O}$ défice de TSH e FSH/LH é comum e pode ser reversível. Os anti-CTLA-4 parecem associar-se a um maior risco de défice hipofisário múltiplo e os anti-PD-1/PD-L1 parecem associar-se mais a défice isolado de ACTH. 2,11,16 Foram também descritos casos raros de diabetes insípida com ipilimumab e avelumab, traduzindo atingimento da hipófise posterior. ${ }^{18,19} \mathrm{O}$ diagnóstico de hipofisite é bioquímico, documentando-se os défices hormonais existentes. A ressonância magnética hipofisária, que não é essencial para o diagnóstico, pode mostrar desde hipófise de aspeto normal até aumento ligeiro/moderado do volume da hipófise, que geralmente normaliza após semanas/meses. O tratamento consiste na substituição hormonal das linhas hipofisárias atingidas: hidrocortisona PO $10-20 \mathrm{mg} /$ dia e levotiroxina (5 - 7 dias após o início da hidrocortisona) (dose inicial $0,8 \mathrm{ug} / \mathrm{kg} / \mathrm{dia}$ ). Pode ser considerada uma dose inicial menor de levotiroxina em doentes idosos ou com doença cardíaca (12,5 - 25 ug/dia), com ajuste posterior de dose às quatro semanas de tratamento, de acordo com o valor de T4 livre. O tratamento do hipogonadismo pode ser considerado em homens ou mulheres em pré-menopausa. O tratamento com corticóides endovenosos em doses elevadas não está recomendado, exceto em caso de crise adrenal ou aumento marcado do volume da hipófise. O seu uso não aumenta a probabilidade de recuperação de função hipofisária nem a sobrevida global dos doentes. ${ }^{2,11,22}$ Após o início do tratamento pode ocorrer recuperação da função hipofisária, nomeadamente do hipotiroidismo e do hipogonadismo, sendo rara a recuperação do 
hipocortisolismo. O tratamento com $\mathrm{ICl}$ não tem que ser suspenso, exceto nos casos de hipofisite grave, em que poderá ser retomado após estabilização da situação clínica. ${ }^{2,11}$ A sua suspensão não altera a probabilidade de recuperação da função hipofisária. ${ }^{2}$ Para diagnóstico precoce recomenda-se vigilância da função tiroideia (TSH e T4 livre) antes do início do tratamento e antes de cada ciclo (ou a cada 4-6 semanas), pelo menos nos primeiros cinco ciclos. ${ }^{2,20}$ Em caso de sintomas sugestivos de hipocortisolismo deve existir um limiar baixo para doseamento de cortisol/ACTH. Em caso de diagnóstico de hipofisite, devem ser doseados $\mathrm{FSH} / \mathrm{LH}$ e estradiol/testosterona para documentação de eventual hipogonadismo. É necessário manter vigilância e suspeição mesmo após suspensão de $\mathrm{ICl}$ (Tabela 2). 2,15,20

\section{Tiroidite}

A tiroidite é a endocrinopatia mais frequente com $\mathrm{ICl}$ e ocorre com maior frequência com os anti-PD-1 (5\% - 10\%), em monoterapia ou associação com anti-CTLA-4.,21 Apesar de descrita como mais rara com os anti-PD-L1, talvez devido à sua aprovação mais recente, um estudo recentemente publicado mostrou uma incidência de $21 \%$ de disfunção tiroideia sob anti-PD-L1.22 A disfunção tiroideia pode ocorrer a qualquer momento após o início do tratamento, mas é mais frequente nas primeiras seis semanas e é mais precoce com a terapêutica combinada. ${ }^{2,22} \mathrm{O}$ mecanismo fisiopatológico não é totalmente conhecido, mas pensa-se que ocorra uma tiroidite destrutiva mediada por células $\mathrm{T}$ citotóxicas. Inicialmente, há uma fase de tireotoxicose (por libertação das hormonas tiroideias armazenadas na tiróide), que pode ser clínica/subclínica e que evolui semanas/ meses depois para eutiroidismo ou hipotiroidismo primário, resultante da destruição da tiróide. ${ }^{2,6}$ Contudo, há doentes que se apresentam logo com hipotiroidismo (subclínico/clínico), que pode ser transitório ou permanente. ${ }^{2} \mathrm{~A}$ etiologia da tiroidite destrutiva não está estabelecida. Foi proposto que, uma vez que a tiróide expressa PD-L1, o bloqueio da via PD-1 possa levar a autoimunidade tiroideia local. ${ }^{23}$ Por outro lado, epítopos tumorais podem possuir sequências de aminoácidos semelhantes a auto-antigénios tiroideus, levando os linfócitos a reagir contra células tiroideias. ${ }^{3}$ Tem sido estudada a relação entre este tipo de tiroidite e os anticorpos anti-peroxidase (anti-TPO) e anti-tiroglobulina (anti-Tg). Apesar da sua incidência parecer ser superior em doentes com anticorpos anti-TPO e anti-Tg prévios positivos, também estão descritos casos com anticorpos positivos apenas após o início do tratamento e identificados doentes que nunca apresentam anticorpos positivos. Além disso, nem sempre a positividade dos anticorpos se associa a disfunção tiroideia. Foi proposto que o aparecimento dos anticorpos após o início do tratamento se possa dever a uma resposta humoral perante uma maior exposição de antigénios tiroideus, provocada por tiroidite destrutiva, ${ }^{2}$ pelo que não está estabelecido se os anticorpos antitiroideus são fator de risco para este tipo de tiroidite..$^{2,3,6,11}$

Tabela 2 - Manifestações endócrinas autoimunes da terapêutica com inibidores do checkpoint

\begin{tabular}{|c|c|c|c|}
\hline Endocrinopatia & $\begin{array}{l}\text { Classe de } \\
\text { inibidor do checkpoint }\end{array}$ & Apresentação & Proposta de protocolo de vigilância \\
\hline \multirow[t]{2}{*}{ Hipofisite } & Anti-CTLA-4 & $\begin{array}{l}\text { Geralmente, défice de } \\
\text { ACTH + défice de TSH e/ } \\
\text { ou FSH+LH }\end{array}$ & $\begin{array}{l}\text { - Antes de cada ciclo de tratamento: do- } \\
\text { sear TSH e T4 livre } \\
\text { - Se sintomas: dosear ACTH e cortisol } \\
\text { matinal }\end{array}$ \\
\hline & $\begin{array}{l}\text { Anti-PD-1 } \\
\text { Anti-PD-L1 }\end{array}$ & $\begin{array}{l}\text { Geralmente, défice isolado } \\
\text { de ACTH }\end{array}$ & $\begin{array}{l}\text { - Se diagnóstico de hipofisite: dosear FSH } \\
\text { e LH + estradiol/testosterona }\end{array}$ \\
\hline Tiroidite & $\begin{array}{l}\text { Principalmente Anti-PD-1 } \\
\text { Anti-PD-L1 }\end{array}$ & $\begin{array}{l}\text { Hiper/hipotiroidismo clínico/ } \\
\text { subclínico }\end{array}$ & $\begin{array}{l}\text { Dosear TSH e T4 livre antes de cada } \\
\text { ciclo de tratamento }\end{array}$ \\
\hline Diabetes & $\begin{array}{l}\text { Principalmente Anti-PD-1 } \\
\text { Anti-PD-L1 }\end{array}$ & $\begin{array}{l}\text { Hiperglicemia aguda e sin- } \\
\text { tomática de novo ou agra- } \\
\text { vamento de diabetes prévia }\end{array}$ & $\begin{array}{l}\text { Medição de glicemia e vigilância de sinais/ } \\
\text { sintomas de hiperglicemia antes de cada } \\
\text { ciclo durante } 12 \text { semanas e depois a cada } \\
3 \text { - } 6 \text { semanas }\end{array}$ \\
\hline $\begin{array}{l}\text { Insuficiência suprarrenal } \\
\text { primária }\end{array}$ & $\begin{array}{l}\text { Anti-CTLA-4, anti-PD-1, } \\
\text { anti-PD-L1 }\end{array}$ & $\begin{array}{l}\text { - Défice de glucocorti- } \\
\text { coides (cortisol normal/ } \\
\text { baixo com elevação de } \\
\text { ACTH) } \\
\text { - Défice de mineralocor- } \\
\text { ticóides (aldosterona } \\
\text { baixa e renina elevada, } \\
\text { hiponatremia e hipercale- } \\
\text { mia) }\end{array}$ & $\begin{array}{l}\text { Se sintomas: dosear ACTH e cortisol } \\
\text { matinal }\end{array}$ \\
\hline Hipoparatiroidismo primário & $\begin{array}{l}\text { Anti-PD-1 } \\
\text { (em monoterapia ou } \\
\text { associação com } \\
\text { anti-CTLA-4) }\end{array}$ & $\begin{array}{l}\text { Hipocalcemia, } \\
\text { hiperfosfatemia, PTH } \\
\text { normal/baixa }\end{array}$ & $\begin{array}{l}\text { Se sintomas: dosear cálcio, fósforo e } \\
\text { PTH }\end{array}$ \\
\hline
\end{tabular}

CTLA-4: antigénio-4 de linfócito T citotóxico; PD-1: proteína de morte celular programada 1; PD-L1: ligando 1 da proteína de morte celular programada 1; ACTH: corticotrofina; TSH: tireotrofina; FSH: hormona folículo-estimulante; LH: hormona luteinizante; T4: tetraiodotironina; PTH: hormona paratiroideia 
A apresentação clínica consiste numa primeira fase de tireotoxicose, geralmente com sintomas inespecíficos e ligeiros. Os mais frequentes são palpitações, emagrecimento, astenia, hipersudorese, diarreia, tremor e intolerância ao calor. ${ }^{2}$ Posteriormente, pode surgir uma fase de hipotiroidismo (subclínico/clínico), também muitas vezes com sintomas inespecíficos e ligeiros, como astenia, aumento de peso, obstipação, bradicardia, intolerância ao frio, pele seca. ${ }^{2} \mathrm{O}$ tempo mediano entre as duas fases é de 4 a 7 semanas. ${ }^{2}$ Apesar desta ser a apresentação típica, qualquer alteração de função tiroideia é possível, independentemente da existência ou não de patologia tiroideia prévia. ${ }^{24} \mathrm{Em}$ doentes com hipotiroidismo primário prévio medicado pode haver alteração da função tiroideia, na maioria com necessidade de aumento de dose de levotiroxina. ${ }^{24} \mathrm{O}$ diagnóstico é bioquímico, com doseamento de TSH e T4 livre. O doseamento de anti-TPO e anti-Tg não deve ser feito por rotina. ${ }^{2}$ A ecografia tiroideia, não necessária para o diagnóstico, pode mostrar achados compatíveis com tiroidite, como estrutura heterogénea ou difusamente hipoecogénica e diminuição da vascularização. ${ }^{2} \mathrm{~A}$ cintigrafia tiroideia mostra diminuição da captação do radiofármaco e apenas é útil para diagnóstico diferencial com doença de Graves. ${ }^{2}$ A PET FDG pode mostrar um aumento difuso da captação, típico de tiroidite inflamatória. ${ }^{2} \mathrm{Na}$ fase tireotóxica, o tratamento tem como objetivo o controlo de sintomas com beta-bloqueantes (ex. propranolol 10 - $30 \mathrm{mg}$, três vezes ao dia). O tratamento com glucocorticóides não está recomendado, exceto em casos de tireotoxicose grave. O tratamento com antitiroideus de síntese não está recomendado. Deve ser monitorizada a função tiroideia a cada 2 - 3 semanas, pois pode haver rápida progressão para hipotiroidismo. 0 hipotiroidismo deve ser tratado com levotiroxina caso seja sintomático (independentemente do valor de TSH) ou, sendo assintomático, com TSH persistentemente acima de 10 $\mathrm{UI} / \mathrm{mL}$ (pelo menos dois doseamentos com quatro semanas de intervalo). A dose inicial de levotiroxina é de $0,8 \mathrm{ug} / \mathrm{kg} /$ dia (12,5 - 25 ug/dia em idosos ou doentes com patologia cardíaca), com titulação de dose a cada 4 - 6 semanas. s,20 $^{2}$ $\mathrm{Na}$ maioria dos doentes não há necessidade de suspender o tratamento com $\mathrm{ICl}^{2}$ Se a disfunção tiroideia for grave, o $\mathrm{ICl}$ deve ser suspenso até haver estabilização clínica. ${ }^{20}$ Para diagnóstico precoce, recomenda-se dosear TSH e T4 livre antes do início do tratamento e antes de cada ciclo (ou a cada 4 a 6 semanas), pelo menos nos primeiros cinco ciclos. ${ }^{2,20}$ Devem fazer-se análises de função tiroideia em qualquer doente (previamente) tratado com ICl e sinais/ sintomas sugestivos de hiper/hipotiroidismo. Os doentes com hipotiroidismo primário previamente medicado devem ser regularmente vigiados pois pode haver necessidade de aumento ou redução da dose de levotiroxina (Tabela 2 ). ${ }^{2}$

\section{Doença de Graves}

Há raros relatos de caso de doença de Graves no contexto de tratamento com ICI (anti-CTLA-4: ipilimumab; anti-PD-1: nivolumab). Foi descrita como um quadro de tireotoxicose isolada (sem orbitopatia), mas também como orbitopatia isolada (sem tireotoxicose), com anticorpos anti-recetor da TSH (TRABs) positivos ou negativos, em qualquer uma das formas de apresentação. ${ }^{2,25}$

\section{Pâncreas endócrino: Checkpoint Inhibitor-Associated Autoimmune Diabetes (CIADM)}

O aparecimento de diabetes mellitus (DM) em contexto de $\mathrm{ICl}$ foi descrito, pela primeira vez, em 2015, com pembrolizumab. ${ }^{26}$ Apesar de ainda ser um evento considerado raro $(0,2 \%-1,0 \%)$, a sua incidência tem vindo a aumentar. ${ }^{27} \mathrm{O}$ mecanismo fisiopatológico é desconhecido, mas pensa-se que ocorra uma destruição direta das células beta pancreáticas pelas células T citotóxicas. ${ }^{28-30}$ Tem sido estudada a relação entre este tipo de DM e haplótipos de risco para diabetes tipo 1 (DM1). Apesar de não haver evidência de uma relação causal, antígenos leucocitários humanos (HLA) de risco para DM1 parecem ser mais prevalentes nestes doentes do que na população em geral. ${ }^{7}$ Não se sabe se os autoanticorpos anti-ilhéus pancreáticos estão envolvidos na patogénese ou se predizem aparecimento deste tipo de DM. ${ }^{2}$ Cerca de metade dos doentes apresentam positividade para, pelo menos, um anticorpo, sendo o anti-GAD65 o mais frequente. Contudo, os restantes doentes não têm positividade identificada para nenhum dos anticorpos conhecidos, o que sugere que a ativação major e súbita de clones de células T CD8+ ocorra sem envolvimento de imunidade humoral. 2,7,31,32 O PD-L1 é expresso nas células de ilhéus pancreáticos e a CIADM ocorre, na maioria dos casos, com anti-PD-1/PD-L1, o que sugere que esta via é importante para a manutenção da auto-tolerância para ilhéus pancreáticos. ${ }^{2,7,33} \mathrm{O}$ tempo de aparecimento após o início do tratamento é variável (uma semana a 12 meses). ${ }^{2,11,31,33}$ A apresentação clínica típica caracteriza-se pelo aparecimento agudo de hiperglicemia grave e sintomática, com rápida progressão para insulinopenia e elevada prevalência de cetoacidose diabética (CAD) e peptídeo $\mathrm{C}$ baixo ao diagnóstico (cerca de $70 \%$ ). ${ }^{2}$ Devido à instalação abrupta do quadro, a hemoglobina glicosilada é proporcionalmente mais baixa do que a glicemia, traduzindo a sua sensibilidade limitada para deteção precoce deste tipo de DM. ${ }^{2,7}$ A CIADM pode também aparecer em doentes com diagnóstico prévio de diabetes tipo 2 (DM2) ou pré-diabetes. Assim, sugere-se um limiar de suspeição clínica baixo perante um agravamento inexplicado do controlo glicémico nestes doentes. ${ }^{16,28}$ Imagiologicamente, o pâncreas pode ter aspetos muito variáveis, desde um aspeto normal, a atrofia ou aumento do volume. ${ }^{2,7}$ A maioria dos doentes evolui para insulinopenia permanente, pelo que o tratamento consiste em insulinoterapia com múltiplas administrações diárias de insulina. Perante uma DM de novo em contexto de $\mathrm{ICI}$, ou agravamento de DM prévia, a insulinoterapia deve ser precocemente iniciada. ${ }^{2,20,28} \mathrm{O}$ tratamento com glucocorticóides não tem benefício na reversão deste tipo de EAir e é contraproducente, por agravar o controlo glicémico. ${ }^{2}$ Em caso de hiperglicemia marcada ou CAD, o tratamento com $\mathrm{ICl}$ deve ser temporariamente suspenso, até que ocorra a estabilização clínica do doente. Para a 
realização de um diagnóstico precoce, recomenda-se monitorizar a glicemia e sinais/sintomas de hiperglicemia antes de iniciar o tratamento e antes de cada ciclo, durante as 12 primeiras semanas de tratamento e depois a cada 3 - 6 semanas. Recomenda-se também a educação dos doentes sobre sinais/sintomas de hiperglicemia e CAD (Tabela 2). $2,11,20,28$

\section{Insuficiência suprarrenal primária}

Raros casos $(0,7 \%)$ de insuficiência suprarrenal primária foram descritos em associação com as três classes de $\mathrm{ICl}^{2,6,17} \mathrm{O}$ mecanismo é desconhecido, presumindo-se que ocorra inflamação e destruição das suprarrenais. ${ }^{2,11}$ Estão descritos casos de doentes com níveis elevados de anticorpos ati-suprarrenal (anticorpos anti-21-hidroxilase e anti-córtex suprarrenal), mas desconhece-se se têm papel na patogénese ou valor prognóstico. ${ }^{2} \mathrm{O}$ tempo de aparecimento após o início do fármaco foi variável (10 semanas com anti-PD-1 e 16 semanas com anti-CTLA-4). ${ }^{11}$ A apresentação é típica de insuficiência suprarrenal primária. ${ }^{2} \mathrm{O}$ diagnóstico é bioquímico, com documentação de défice de glucocorticoides (cortisol normal/baixo com elevação de $\mathrm{ACTH}$ ) e mineralocorticóides (aldosterona baixa e renina elevada, hiponatremia e hipercalemia). ${ }^{2}$ Imagiologicamente, pode haver evidência de inflamação com aumento bilateral difuso e margens regulares das suprarrenais, mas estas podem ter aspeto normal ou sinais de atrofia. ${ }^{2} \mathrm{~A}$ PET-FDG pode mostrar hipercaptação difusa bilateral das suprarrenais. É importante o diagnóstico diferencial com outras causas de insuficiência suprarrenal primária, tais como metastização ou hemorragia bilateral. ${ }^{2} \mathrm{O}$ tratamento consiste na reposição de gluco e mineralocorticóides. Se a apresentação clínica for ligeira ou moderada, os doentes poderão ser tratados em ambulatório com $10-20 \mathrm{mg} /$ dia de hidrocortisona e 0,05 - 2,0 mg/dia de fludrocortisona. ${ }^{2}$ Em caso de doença grave, devem ser internados e fazer tratamento inicial endovenoso. Nesta última situação, o tratamento com $\mathrm{ICl}$ deve ser temporariamente suspenso até à estabilização clínica ${ }^{11} \mathrm{~A}$ maioria dos doentes permanece com insuficiência suprarrenal, com necessidade de tratamento definitivo (Tabela 2)..$^{2,11}$

\section{Hipoparatiroidismo primário}

Foram descritos quatro casos de hipoparatiroidismo primário por $\mathrm{ICl}$, todos com sintomas neuromusculares de hipocalcemia aguda grave ao diagnóstico. ${ }^{34-37}$ Estes doentes estavam tratados com anti-PD-1 (dois em monoterapia e dois em combinação). O hipoparatiroidismo ocorreu um a quatro meses após o início do tratamento e todos os doentes ficaram com hipoparatiroidismo primário definitivo. ${ }^{34-37}$

\section{REFERÊNCIAS}

1. Pardoll DM. The blockade of immune checkpoints in cancer immunotherapy. Nat Rev Cancer. 2012;12:252-64.

2. Chang LS, Barroso-Sousa R, Tolaney SM, Hodi FS, Kaiser UB, Min L. Endocrine toxicity of cancer immunotherapy targeting immune checkpoints. Endocr Rev. 2019;40:17-65.
Desconhece-se o mecanismo subjacente: poderá dever-se a destruição imuno-mediada das paratiroides (hipoparatiroidismo permanente) ou a hiperativação do calcium-sensor receptor (CaSR) por anticorpos estimuladores. Estes anticorpos inibem a secreção de paratormona (PTH), mantendo o cálcio abaixo do limiar que estimularia a sua libertação (hipoparatiroidismo funcional). ${ }^{35-36} \mathrm{O}$ diagnóstico é bioquímico, com evidência de hipocalcemia, hiperfosfatemia e PTH normal ou baixa. O tratamento consiste na toma de cálcio e de formas ativas de vitamina $\mathrm{D}$, para um objetivo de cálcio sérico na ordem dos $7,5-8,5 \mathrm{~mL} / \mathrm{dL}$. ${ }^{34-}$ ${ }^{37}$ Apesar da raridade de casos, uma metanálise sobre alterações eletrolíticas com $\mathrm{ICl}$ encontrou uma associação significativa entre o tratamento com anti-PD-1 e o risco de hipocalcemia (RR 10,87; IC 95\% 1,4 - 84,16). ${ }^{38}$ Assim, nomeadamente com anti-PD-1, a incidência de hipocalcemia ligeira ou assintomática, e eventualmente transitória, poderá ser mais elevada do que o descrito (Tabela 2).

\section{CONCLUSÃO}

Nos próximos anos, perspetiva-se uma utilização crescente de $\mathrm{ICl}$ no tratamento de muitas neoplasias. As endocrinopatias associadas a $\mathrm{ICl}$ possuem um espectro clínico muito variável, podendo haver atingimento simultâneo/ sequencial uni/pluriglandular e défices hormonais isolados/ múltiplos. Os seus mecanismos fisiopatológicos e fatores de risco permanecem por esclarecer, assim como a sua relação com o prognóstico da doença oncológica. Contrariamente a eventos adversos autoimunes noutros órgãos, as endocrinopatias geralmente não implicam a suspensão de $\mathrm{ICl}$, são maioritariamente irreversíveis e a utilização de glucocorticóides não tem utilidade na sua reversão. A imprevisibilidade da sua ocorrência ou do tempo decorrido após o início do tratamento obrigam a uma vigilância regular dos doentes. Apesar de tratáveis, o maior desafio passa pelo seu diagnóstico e início de tratamento atempados, reduzindo a sua morbilidade e mortalidade. Isto obrigará a uma abordagem multidisciplinar, com participação das várias especialidades médicas envolvidas no tratamento dos doentes oncológicos, em ambulatório, no serviço de urgência ou em internamento hospitalar.

\section{CONFLITOS DE INTERESSE}

A autora declara não ter conflitos de interesses relacionados com o presente trabalho.

\section{FONTES DE FINANCIAMENTO}

Este trabalho não recebeu qualquer tipo de suporte financeiro de nenhuma entidade no domínio público ou privado. 
4. U. S. Food \& Drug Administration. Drugs@FDA: FDA-Approved Drugs. [consultado 2020 fev 28]. Disponivel em: https://www.accessdata.fda. gov/scripts/cder/daf/index.cfm.

5. Ferrari SM, Fallahi P, Elia G, Ragusa F, Ruffilli I, Patrizio A et al. Autoimmune endocrine dysfunctions associated with cancer immunotherapies. Int J Mol Sci. 2019;20:2560

6. Barroso-Sousa R, Barry WT, Garrido-Castro AC, Hodi FS, Min L, Krop IE, et al. Incidence of endocrine dysfunction following the use of different immune checkpoint inhibitor regimens: a systematic review and metaanalysis. JAMA Oncol. 2018;4:173-82

7. de Filette JM, Pen JJ, Decoster L, Vissers T, Bravenboer B, Van der Auwera BJ, et al. Immune checkpoint inhibitors and type 1 diabetes mellitus: a case report and systematic review. Eur J Endocrinol. 2019;181:363-74

8. Zhai $Y, Y e X, H u F, X u J$, Guo $X$, Zhuang $Y$, et al. Endocrine toxicity of immune checkpoint inhibitors: a real-world study leveraging US Food and Drug Administration adverse events reporting system. J Immunother Cancer. 2019;7:286.

9. Tan MH, lyengar R, Mizokami-Stout K, Yentz S, MacEachern MP, Shen LY, et al. Spectrum of immune checkpoint inhibitors-induced endocrinopathies in cancer patients: a scoping review of case reports. Clin Diabetes Endocrinol. 2019;22;5:1

10. Kassi E, Angelousi A, Asonitis N, Diamantopoulos P, Anastasopoulou A, Papaxoinis G, et al. Endocrine-related adverse events associated with immune-checkpoint inhibitors in patients with melanoma. Cancer Med. 2019;8:6585-94

11. Arima $\mathrm{H}$, Iwama $\mathrm{S}$, Inaba $\mathrm{H}$, Ariyasu $\mathrm{H}$, Makita $\mathrm{N}$, Otsuki $\mathrm{M}$, et al. Management of immune-related adverse events in endocrine organs induced by immune checkpoint inhibitors: clinical guidelines of the Japan Endocrine Society. Endocr J. 2019;66:581-6.

12. Lu J, Li L, Lan $Y$, Liang $Y$, Meng H. Immune checkpoint inhibitorassociated pituitary-adrenal dysfunction: A systematic review and metaanalysis. Cancer Med. 2019;8:7503-15.

13. Garon-Czmil J, Petitpain N, Rouby F, Sassier M, Babai S, YéléhéOkouma M, et al. Immune check point inhibitors-induced hypophysitis: a retrospective analysis of the French Pharmacovigilance database. Sci Rep. 2019;9:19419.

14. Caturegli P, Di Dalmazi G, Lombardi M, Grosso F, Larman HB, Larman $\mathrm{T}$, et al. Hypophysitis secondary to cytotoxic T-lymphocyte-associated protein 4 blockade: insights into pathogenesis from an autopsy series. Am J Pathol. 2016;186:3225-35.

15. Thapi S, Leiter A, Galsky M, Gallagher EJ. Recovery from secondary adrenal insufficiency in a patient with immune checkpoint inhibitor therapy induced hypophysitis. J Immunother Cancer. 2019;7:248.

16. Ohara N, Kobayashi M, Ikeda Y, Hoshi T, Morita S, Kanefuji T, et al. Non-insulin-dependent diabetes mellitus induced by immune checkpoint inhibitor therapy in an insulinoma-associated antigen-2 autoantibodypositive patient with advanced gastric cancer. Intern Med. 2020;59:5516.

17. Lanzolla G, Coppelli A, Cosottini M, Del Prato S, Marcocci C, Lupi I. Immune checkpoint blockade anti-PD-L1 as a trigger for autoimmune polyendocrine syndrome. J Endocr Soc. 2019;3:496-503.

18. Hamnvik OP, Laury AR, Laws Jr ER, Kaiser UB. Lymphocytic hypophysitis with diabetes insipidus in a young man. Nat Rev Endocrinol. 2010;6:464-70

19. Zhao C, Tella SH, Del Rivero J, Kommalapati A, Ebenuwa I, Gulley J, et al. Anti-PD-L1 treatment induced central diabetes insipidus. J Clin Endocrinol Metab. 2018:103:365-9.

20. Brahmer JR, Lacchetti C, Schneider BJ, Atkins MB, Brassil KJ, Caterino JM, et al. Management of immune-related adverse events in patients treated with immune checkpoint inhibitor therapy: American Society of Clinical Oncology Clinical Practice Guideline. J Clin Oncol. 2018:36:1714-68.

21. Al Mushref M, Guido PA, Collichio FA, Moore DT, Clemmons DR. Thyroid dysfunction, recovery, and prognosis in melanoma patients treated with immune checkpoint inhibitors: a retrospective review. Endocr Pract. 2020;26:36-42.

22. Kotwal A, Kottschade L, Ryder M. PD-L1 inhibitor-induced thyroiditis is associated with better overall survival in cancer patients. Thyroid. 2020;30:177-84

23. Yamauchi I, Sakane Y, Fukuda Y, Fujii T, Taura D, Hirata M, et al. Clinical features of nivolumab-induced thyroiditis: a case series study. Thyroid. 2017;27:894-901.

24. Patel NS, Oury A, Daniels GA, Bazhenova L, Patel SP. Incidence of thyroid function test abnormalities in patients receiving immunecheckpoint inhibitors for cancer treatment. Oncologist. 2018;23:123641.

25. Campredon P, Imbert P, Mouly C, Grunenwald S, Mazières J, Caron P. Severe inflammatory ophthalmopathy in a euthyroid patient during nivolumab treatment. Eur Thyroid J. 2018;7:84-7.

26. Hughes J, Vudattu N, Sznol M, Gettinger S, Kluger H, Lupsa B, et al. Precipitation of autoimmune diabetes with anti-PD-1 immunotherapy. Diabetes Care. 2015;38:e55-7.

27. Wright JJ, Salem JE, Johnson DB, Lebrun-Vignes B, Stamatouli A, Thomas JW, et al. Increased reporting of immune checkpoint inhibitorassociated diabetes. Diabetes Care. 2018;41:e150-1.

28. Alrifai T, Ali FS, Saleem S, Ruiz DC, Rifai D, Younas S, et al. Immune checkpoint inhibitor induced diabetes mellitus treated with insulin and metformin: evolution of diabetes management in the era of immunotherapy. Case Rep Oncol Med. 2019;2019:8781347.

29. Kotwal A, Haddox C, Block M, Kudva YC. Immune checkpoint inhibitors: an emerging cause of insulin-dependent diabetes. BMJ Open Diabetes Res Care. 2019;7:e000591.

30. Tsang VH, McGrath RT, Clifton-Bligh RJ, Scolyer RA, Jakrot V, Guminsk $A D$, et al. Checkpoint inhibitor-associated autoimmune diabetes is distinct from type 1 diabetes. J Clin Endocrinol Metab. 2019;104:5499506.

31. Zezza M, Kosinski C, Mekoguem C, Marino L, Chtioui H, Pitteloud N, et al. Combined immune checkpoint inhibitor therapy with nivolumab and ipilimumab causing acute-onset type 1 diabetes mellitus following a single administration: two case reports. BMC Endocr Disord. 2019;19:144.

32. Gauci ML, Boudou P, Baroudjian B, Vidal-Trecan T, Da Meda L, Madelaine-Chambrin I, et al. Occurrence of type 1 and type 2 diabetes in patients treated with immunotherapy (anti-PD-1 and/or anti-CTLA-4) for metastatic melanoma: a retrospective study. Cancer Immunol Immunother. 2018;67:1197-208.

33. Sothornwit J, Phunmanee A, Pongchaiyakul C. Atezolizumab-induced autoimmune Diabetes in a Patient With Metastatic Lung Cancer. Front Endocrinol (Lausanne). 2019;10:352.

34. Win MA, Thein KZ, QdaisatA, Yeung SJ. Acute symptomatic hypocalcemia from immune checkpoint therapy-induced hypoparathyroidism. Am J Emerg Med. 2017;35:1039.e5-7.

35. Trinh B, Sanchez GO, Herzig P, Läubli H. Inflammation-induced hypoparathyroidism triggered by combination immune checkpoint blockade for melanoma. J Immunother Cancer. 2019;7:52

36. Piranavan P, Li Y, Brown E, Kemp EH, Trivedi N. Immune checkpoint inhibitor-induced hypoparathyroidism associated with calciumsensing receptor-activating autoantibodies. J Clin Endocrinol Metab. 2019:104:550-6.

37. Umeguchi $H$, Takenoshita $H$, Inoue $H$, Kurihara $Y$, Sakaguchi $C$, Yano $S$, et al. Autoimmune-related primary hypoparathyroidism possibly induced by the administration of pembrolizumab: a case report. J Oncol Pract. 2018;14:449-51.

38. Manohar S, Kompotiatis P, Thongprayoon C, Cheungpasitporn W, Herrmann J, Herrmann SM. Programmed cell death protein 1 inhibitor treatment is associated with acute kidney injury and hypocalcemia: meta-analysis. Nephrol Dial Transplant. 2019;34:108-17. 